\title{
Application of renewable energy sources in hospital services
}

\author{
${ }^{a}$ Carlos Alberto de León Benítez, ${ }^{b}$ Ernesto Santiago Garmendia López \\ ${ }^{a}$ Profesor Titular, Dr. CT. Centro de Estudios Electroenergéticos. Universidad Central "Marta Abreu" de Las Villas. \\ e-mail: charle@uclv.edu.cu \\ ${ }^{b}$ Profesor adjunto, M.Sc. Universidad Central "Marta Abreu” de Las Villas. \\ e-mail: egarmendia@uclv.cu
}

\begin{abstract}
The proposal of a thermal solar system to heat water in the Hospital service is the fundamental objective of the present work, which had the aim of carry out a study on the employment of renewable energy sources to increase the energy efficiency and the quality of the hospitable service. The methodology used to select the proposed system took in consideration the rules of thumbs offered by several international organizations as RETScreen, SODEANS and Swisscontact, different computer software were employed. This project, with an approximate initial cost of $\$ 37$ 200, allows to obtain an annual electric energy saving of 190 MW.h and it contribute a net annual greenhouse gas emission reduction equivalent to 440 barrels of crude oil not consumed.
\end{abstract}

Keywords: Solar heater, sun energy, renewable energy, sun collectors.

\section{Aplicación de fuentes renovables de energía en servicios hospitalarios}

\section{RESUMEN}

La propuesta de un sistema solar térmico para el calentamiento de agua en servicios hospitalarios es el objetivo fundamental del presente trabajo, el cual tuvo como fin realizar un estudio sobre el empleo de fuentes renovables de energía para aumentar su eficiencia y la calidad del servicio. La metodología seguida para seleccionar el sistema propuesto tomó en consideración las reglas prácticas brindadas por organizaciones internacionales como RETScreen, SODEAN y Swisscontact, empleándose diferentes programas de computación. Se incluye un ejemplo de aplicación en una instalación hospitalaria de la ciudad de Santa Clara, Cuba. Este proyecto, con una inversión inicial de \$37200, permite obtener anualmente un ahorro de energía eléctrica de 190 MW.h y aporta una reducción neta en la emisión de gases de efecto invernadero equivalente a dejar de consumir 440 barriles de petróleo crudo.

Palabras claves: Calentadores solares, energía solar, energía renovable, colectores solares.

\section{Introdución}

El presente trabajo se desarrolló en el Hospital Pediátrico Docente "José Luis Miranda" ubicado en la ciudad de Santa Clara, provincia de Villa Clara, en él se analiza la posibilidad de emplear fuentes renovables de energía (FRE) con vista a mejorar la calidad del servicio hospitalario y el nivel de satisfacción de los pacientes ingresados, aumentando a su vez la eficiencia energética de la institución y la protección al medio ambiente. En particular se realiza el diseño de un sistema de calentamiento de agua mediante colectores solares comercializados en Cuba.

La realización del mismo está vinculada a los esfuerzos del país por elevar la eficiencia de sus empresas como parte de la política económica seguida en la actualidad y a la necesidad de emprender acciones encaminadas a la protección del medio ambiente.

El empleo de las FRE data desde el comienzo mismo de la humanidad con el uso de biomasas para cocinar los alimentos, protegerse del frío y de diferentes animales; luego aprovecharon la energía eólica para propulsar las embarcaciones, llegando a utilizarla en los molinos de viento y hasta para irrigar los campos cultivados. "Se tienen referencias sobre proyectos del emperador Hammurabi para irrigación, empleando la energía eólica, que data del 1700 A.C. Posteriormente los persas, los chinos, los holandeses, etc., emplearon con éxito a través de los siglos la 
Carlos A. de León Benítez, \& Ernesto Santiago Garmendia López/ITEGAM-JETIA Vol.01, Nº 02, pp.17-21. Junho, 2015.

energía del viento. En la actualidad existen aún en operación algunos centenares de aerobombas del tipo multipala, del cual se fabricaron más de seis millones, después de su invención en 1854'[1].

El descubrimiento de grandes yacimientos de combustible fósiles con el consecuente perfeccionamiento de una tecnología más sencilla para su explotación causó la decadencia de los pequeños sistemas descentralizados de aprovechamiento de las FRE y el establecimiento en su lugar de sistemas altamente centralizados, que si bien ofrecían energía abundante y barata para el desarrollo industrial, trajeron consigo la implantación de patrones de consumo irracional y el deterioro del medio ambiente.

En la actualidad se han desarrollado sistemas confiables, eficaces y altamente rentables desde el punto de vista económico basados en FRE y se ha tomando conciencia del importante papel jugado por la variable ecológica, al reconocerse que de continuar la quema acelerada de combustibles fósiles se producirían catástrofes mundiales poniendo en peligro de extinción a la humanidad. El continuo aumento detectado en los niveles de $\mathrm{CO}_{2}$ en la atmósfera y la destrucción de su capa de ozono son algunos de los fenómenos que más alarman a los científicos.

Los análisis realizados en varios hospitales, tanto en Cuba como en el resto del mundo, ponen de manifiesto el insuficiente nivel de gestión energética existente en muchos de ellos, así como las posibilidades de reducir los consumos y costos energéticos mediante la creación de las capacidades técnico-organizativas para administrar eficientemente la energía [2].

La revolución energética cubana actual hace énfasis en el empleo de FRE estables y económicas, particularmente los hospitales presentan la necesidad de garantizar agua caliente a temperatura adecuada para los diferentes servicios, lo cual está confrontando dificultades. En la revista electrónica "Ciencias Holguín" [3], se cita como una fuente capaz de resolver este problema el uso de la energía solar, la cual presenta grandes ventajas.

Por tanto este trabajo realiza una propuesta tecnológica a partir del uso de fuentes renovables de energía, para el mejoramiento de la gestión energética en el Hospital Pediátrico Docente "José Luis Miranda" a la vez que contribuye al aumento de la calidad del servicio hospitalario y el nivel de satisfacción de los pacientes.

\section{Análisis del posible empleo de energía solar térmica en el Hospital Infantil}

Consiste en el aprovechamiento de la energía solar desde el punto de vista térmico; en la actualidad se siguen dos variantes fundamentales en su aprovechamiento:

a) La energía solar térmica pasiva. Se aplica casi exclusivamente en las nuevas construcciones siguiendo el concepto de la arquitectura bioclimática, no precisa de elementos mecánicos al aprovechar directamente la luz solar.

b) La energía solar térmica activa. Basada en el calentamiento, mediante la energía solar, de determinado fluido empleado como portador energético, dicha energía es trasferida al medio de trabajo (generalmente agua) y empleada principalmente en el calentamiento de agua sanitaria o en la calefacción de habitaciones.

El uso de la energía solar para calentar agua no es una idea nueva, hace más de 100 años en varios países se emplearon tanques de agua pintados de negro como simples calentadores solares, sin embargo la tecnología del calentamiento solar de agua se mejoró durante el siglo pasado, "hoy el mundo cuenta con cientos de miles de calentadores solares modernos.... De hecho en algunos países la ley actual requiere la instalación de calentadores solares en cada nuevo proyecto de construcción residencial" [8].

El empleo de sistemas solares térmicos presenta grandes ventajas [3], entre las que se encuentran: la no emisión de gases contaminantes perjudiciales para la salud, ni gases de efecto invernadero; no produce desperdicios ni residuos peligrosos; son sistemas silenciosos y confiables que promueven el autoconsumo y la independencia energética; constituye una inversión de rápida recuperación basada en el ahorro de energía convencional; no requiere de trabajos de extracción, transporte o almacenamiento; posee elevada fiabilidad con bajos costos de mantenimiento y emplea un tipo de energía sin riesgo de agotamiento.

Actualmente el hospital infantil no ofrece agua caliente a los pacientes, cada uno de ellos tiene que obtenerla por sus propios medios, lo que implica que el sistema actual de calentamiento de agua (al que se le denomina en lo adelante "caso base" en contraposición al "caso propuesto" que empleará un sistema conformado por calentadores solares) esté formado por calentadores y hornillas traídos por los pacientes, los cuales suelen ser fabricados tanto industrial como artesanalmente. Esta situación hace posible el empleo de calentadores solares de agua de forma económica.

\subsection{Sistema solar térmico propuesto \\ 1) Parámetros de partida.}

La alineación de los colectores solares se realiza a partir de sus ángulos de orientación $(\gamma)$ y de inclinación $(\beta)$, para ello hay que estudiar la irradiación global mensual incidente en su superficie para el lugar donde se van a ubicar y tener en cuenta otros aspectos bien detallados en la referencia [9]. Generalmente se emplea una alineación fija, aunque algunos proyectos proponen el empleo de seguidores automáticos para aumentar la energía captada, pero encarecen el sistema [10]. Como resultados se sugiere emplear una inclinación de $30^{\circ}$ con orientación sur.

Demanda de agua caliente sanitaria. Internacionalmente se acepta para los hospitales una norma de 80 litros diarios de agua a $45^{\circ} \mathrm{C}$ por paciente [11], lo cual se tendrá en cuenta para el caso base, sin embargo es posible adoptar medidas de eficiencia energética para disminuir su consumo en un $10 \%$. Teniendo en cuenta el número de camas (300) y un por ciento ocupacional del $100 \%$ se obtiene una demanda de agua anual de $8760 \mathrm{t}$ de agua a $45^{\circ} \mathrm{C}$ equivalente a 4920 t a $60^{\circ} \mathrm{C}$ que entregará el sistema colector internamente antes de la válvula mezcladora.

Debe establecerse una diferencia entre el agua entregada por el sistema solar térmico al consumo y la generada internamente. El agua entrega a los consumidores debe tener una temperatura de $45^{\circ} \mathrm{C}$ y se obtiene a la salida de una válvula mezcladora de dos entradas, una es el agua de suministro de la red y la otra es el 
Carlos A. de León Benítez, \& Ernesto Santiago Garmendia López/ITEGAM-JETIA Vol.01, Nº 02, pp.17-21. Junho, 2015.

agua caliente entregada por el tanque colector del sistema solar, para la cual se establece en el presente trabajo una temperatura de $60^{\circ} \mathrm{C}$, lo suficientemente alta para evitar la proliferación de microorganismos patógenos y lo más baja posible para disminuir las pérdidas energéticas por transferencia de calor al medio.

Según las normas existen otros requerimientos de agua caliente en la cocina y la lavandería, en el presente trabajo se propone abastecerlos a partir del generador de vapor que alimenta estas dependencias.

La energía térmica (expresada en kJ) se calcula a partir de la "Erro! Fonte de referência não encontrada.", sobre una base mensual, a partir del salto térmico $(\Delta \mathrm{T}$, expresado en Kelvin) y la masa de agua requerida ( $\mathrm{m}$, en $\mathrm{kg}$ ), teniendo el cuidado de emplear la capacidad calórica (cp) en $\mathrm{kJ} /(\mathrm{kg} . \mathrm{K})$.

$$
\text { Energía }=m \cdot c p \cdot \Delta T \quad(k J)
$$

El cálculo de la energía consumida por el sistema encargado de calentar el agua (sistema eléctrico para el caso base y sistema solar térmico para el caso propuesto) debe tener en cuenta su eficiencia. El resumen de las características del caso base se muestra en la "Tabla 1".

Tabla 1: Resumen del sistema de ACS para el caso base.

\begin{tabular}{|c|c|c|}
\hline \multicolumn{3}{|c|}{ Sistema de agua caliente del caso base } \\
\hline Parámetro & UM & Cantidad \\
\hline Demanda de calentamiento total & MW.h & 195.6 \\
\hline Carga de calentamiento punta total & $\mathrm{kW}$ & 37 \\
\hline Horas equivalentes a plena carga & $\mathrm{h}$ & 5185 \\
\hline Eficiencia del sistema & $\%$ & 80 \\
\hline Consumo de electricidad anual & $\mathrm{MW} . \mathrm{h}$ & $246 \mathrm{Don}$ \\
\hline Precio de la electricidad & $\$ / \mathrm{kWh}$ & $0.170 \mathrm{Ci}: \mathrm{Ca}$ \\
\hline Costo de la electricidad & $\$$ & $41760 \mathrm{Aa}$ \\
\hline
\end{tabular}
2.2 Cálculo del sistema propuesto.

Generalmente los sistemas solares térmicos no se diseñan para cubrir toda la demanda debido a que la disponibilidad de su fuente primaria de energía (el sol) es variable, de lo contrario se arribaría a diseños de sistemas solares exageradamente grandes y costosos, ver referencia [12].

El sistema propuesto presenta un circuito solar abierto con circulación forzada, consta de una batería de colectores solares de tubos al vacío, ensamblados en Cuba, con dos tanques donde se acumula el agua caliente proveniente de los colectores, la cual se hace circular mediante una electrobomba. En el tanque de salida se instala un sistema eléctrico de respaldo para estabilizar la temperatura del agua suministrada a los usuarios. Ambos tanques deben estar aislados térmicamente.

La interconexión hidráulica del sistema solar (desde la entrada de los colectores hasta la salida de la válvula mezcladora) puede realizarse con tubería de cobre, acorde al criterio de la referencia [13].

En el análisis del proyecto se empleó una metodología similar a la descrita en la referencia [14], pero con auxilio de los programas de computación SWH3 y RETScreen 4 (referencias [15] y [8]).

En el diseño se emplearon los colectores solares chinos ensamblados en Cuba modelo LPC 47-1530.
Las principales características del caso propuesto se resumen en la tabla "Tabla 2".

Tabla 2: Principales características del sistema solar propuesto.

\begin{tabular}{|c|c|c|}
\hline Parámetro & UM & Cantidad \\
\hline Número de colectores & & 32 \\
\hline Área total de colectores solares & $\mathrm{m}^{2}$ & 198.4 \\
\hline Área total de captación & $\mathrm{m}^{2}$ & 120 \\
\hline Capacidad de generación & $\mathrm{kW}$ & 84 \\
\hline Demanda anual de calor & MW.h & 196.5 \\
\hline Fracción solar & $\%$ & 72.6 \\
\hline Calentamiento anual entregado & MW.h & 142.7 \\
\hline Consumo anual de electricidad & MW.h & 53.8 \\
\hline
\end{tabular}

El sistema de calentamiento solar de agua puede ser ubicado en el techo del edificio de cuatro pisos en el que se encuentran las salas de respiratorio, donde se dispone de un área aproximada de $600 \mathrm{~m}^{2}$ (el área bruta total de colectores requerida es de alrededor de $200 \mathrm{~m}^{2}$ ). Desde aquí puede distribuirse por gravedad el agua caliente a todo el hospital y abastecer el sistema desde el tanque principal ubicado por encima de este nivel.

La capacidad instalada de los sistemas solares de calentamiento de agua, empleada en sustitución del área de colectores para comparar los diferentes sistemas solares térmicos entre sí y con otras fuentes de energía, se expresa en $\mathrm{kW}$ térmicos $\left(\mathrm{kW}_{\mathrm{th}}\right)$ y se determina a través de la siguiente expresión:

$$
C i=A a \cdot N c \times F c
$$

Donde:

$\mathrm{Ci}$ : Capacidad instalada en $\mathrm{kW}$ térmicos.

$A a$ : Área de apertura en $\mathrm{m}^{2}$.

$N c$ : Numero de colectores.

$F c$ : Factor de conversión $\mathrm{kW}_{\mathrm{th}} / \mathrm{m}^{2}$.

Se emplea un factor de conversión de $0,7 \mathrm{~kW}_{\mathrm{th}} / \mathrm{m}^{2}$. ( $\mathrm{kW}$ térmico por $\mathrm{m}^{2}$ de área de apertura de los colectores). Este factor se usa uniformemente para los colectores solares planos, concentrados y de tubos al vacío (Recomendación de la "International Energy Agency's Solar Heating and Cooling Programmer" IEA SHC, 2004).

La bomba de recirculación del circuito solar debe manejar un flujo de $40 \mathrm{~L} / \mathrm{h}$ por $\mathrm{m}^{2}$ de área de captación (recomendado en las referencias [12] y [14]) y teniendo en cuenta que la temperatura del agua puede llegar a superar los $60^{\circ} \mathrm{C}$, se seleccionó una bomba Scanpump serie NR 50/32-16 mediante el empleo del programa de computación "ABSEL".

La citada bomba debe ser gobernada por un dispositivo electrónico denominado "control diferencial de temperatura" que arranca o detiene la bomba en función de la diferencia de temperatura prefijada entre los terminales de la batería de colectores (es decir, entre la salida de la batería de captadores y la salida del depósito acumulador solar hacia los colectores). La referencia [11] propone que "En ningún caso las bombas estarán en marcha con diferencias de temperaturas menores de $2^{\circ} \mathrm{C} n i$ paradas con diferencias superiores a $7^{\circ} \mathrm{C}$ ". Por tanto se sugiere 
detener la bomba con $2^{\circ} \mathrm{C}$, pero arrancarla con $5^{\circ} \mathrm{C}$ como sugiere la referencia [16].

Acumulador solar. Con el objetivo de determinar la capacidad del depósito solar se empleó la razón de "capacidad de almacenamiento vs área de captación" de $75 \mathrm{~L} / \mathrm{m}^{2}$ propuesta por RETScreen. Así se obtuvo una capacidad de 9000 L. Se propone el empleo de dos tanques aislados térmicamente y con cierre hermético de 4 y $5 \mathrm{~m}^{3}$ de capacidad respectivamente. Los tanques pueden ser conectados en serie invertida con el circuito de consumo.

Como sistema auxiliar se propone un calentador eléctrico ubicado en la parte superior (más exactamente en la tercera parte superior) del tanque de $4 \mathrm{~m}^{3}$, dispuesto a la salida del sistema acumulador solar.

Siguiendo el criterio de la referencia [11], donde se sugiere emplear una relación cercana a $150 \mathrm{~W} / \mathrm{m}^{2}$ de superficie de captación, se propone emplear dos resistencias de $13 \mathrm{~kW}$.

En forma simplificada el sistema solar térmico propuesto se muestra en la "Figura 1".

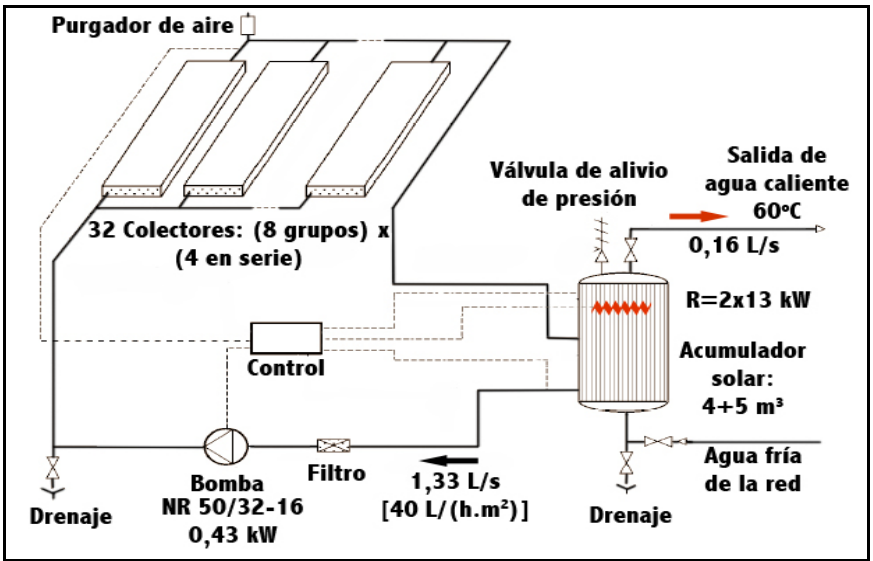

Figura 1: Esquema principal del caso propuesto.

\subsection{Contribución al medio ambiente.}

La implementación del sistema propuesto puede realizar una importante contribución a la protección del medio ambiente al disminuir las emisiones de gases de efecto invernadero, observe la "Erro! Fonte de referência não encontrada.".

Tabla 3: Análisis de la emisión de gases de efecto invernadero.

\begin{tabular}{|c|c|r|}
\hline \multicolumn{2}{|c|}{ Emisión de gases de efecto invernadero (GEI) } \\
\hline $\begin{array}{c}\text { Tipo de } \\
\text { combustible }\end{array}$ & Mezcla de combustible & Emisiones GEI \\
\hline & $\%$ & $\mathrm{t}_{\mathrm{CO} 2}$ \\
\hline \multicolumn{3}{|c|}{ Caso base } \\
\hline Electricidad & 100 & 275 \\
\hline Total & 100 & 275 \\
\hline \multicolumn{3}{|c|}{ Caso propuesto } \\
\hline Solar & 28 & 02 \\
\hline Electricidad & 100 & 62 \\
\hline \multicolumn{2}{|c|}{ Total } & 213 \\
\hline Reducción de emisiones GEI anual neta & \\
\hline
\end{tabular}

La disminución de la emisión de gases de efecto invernadero es equivalente a dejar de consumir 442 barriles de petróleo crudo anual.

\section{Resumen financiero}

A continuación se exponen ("Erro! Fonte de referência não encontrada." y "Tabla 5") algunos indicadores financieros del proyecto y un flujo de caja en el que se muestran sólo 10 de los 20 años de vida útil.

Tabla 4: Resumen de costos, ingresos y viabilidad financiera del proyecto

\begin{tabular}{|c|c|c|}
\hline Resumen y viabilidad financiera & UM & Cantidad \\
\hline Costos iniciales totales & $\$$ & 37192 \\
\hline Costos anuales totales & $\$$ & 9686 \\
\hline Total de ahorros anuales & & 41763 \\
\hline TIR & $\%$ & 91.7 \\
\hline Pago simple de retorno del capital & año & 1.2 \\
\hline Valor presente neto (VPN) & $\$$ & 89300 \\
\hline Relación costo/beneficio & & 3.4 \\
\hline
\end{tabular}

Tabla 5: Flujo de cajas del proyecto

\begin{tabular}{|c|c|c|c|}
\hline Año & $\begin{array}{c}\text { Antes- } \\
\text { impuestos } \\
(\$)\end{array}$ & $\begin{array}{c}\text { Después-impuestos } \\
(\$)\end{array}$ & $\begin{array}{c}\text { Acumulado } \\
(\$)\end{array}$ \\
\hline 0 & -37192 & -35704 & -35704 \\
\hline 1 & 33042 & 27326 & -8378 \\
\hline 2 & 34036 & 28746 & 20368 \\
\hline 3 & 35059 & 29110 & 49478 \\
\hline 4 & 36113 & 29634 & 79112 \\
\hline 5 & 37199 & 30280 & 109392 \\
\hline 6 & 38317 & 31018 & 140410 \\
\hline 7 & 39469 & 31831 & 172240 \\
\hline 8 & 40656 & 32703 & 204944 \\
\hline
\end{tabular}

\section{Conclusiones.}

La energía solar térmica es la fuente de energía renovable con mayor potencialidad de empleo en el Hospital Provincial Pediátrico Docente "José Luis Miranda", es viable económicamente y contribuye a la protección del medio ambiente al reducir el consumo de combustibles fósiles. $\mathrm{Su}$ empleo favorece la elevación del confort de sus pacientes y la calidad del servicio hospitalario a la vez que representa un ahorro económico para el país.

Otras fuentes renovables de energía como la solar fotovoltaica y la biomasa pudieran ser empleadas en un futuro, pero no son las más recomendables con las condiciones económicas actuales del país. 
Carlos A. de León Benítez, \& Ernesto Santiago Garmendia López/ITEGAM-JETIA Vol.01, Nº 02, pp.17-21. Junho, 2015.

El sistema solar térmico propuesto para el calentamiento de agua sanitaria, consta de 32 colectores solares de tubos al vacío fabricados en Cuba. Este sistema solar, con una inversión inicial aproximada de $\$ 37200$, permite obtener un ahorro de energía eléctrica anual de 190 MWh y aporta una contribución a la reducción de gases de efecto invernadero equivalente a dejar de consumir 440 barriles de petróleo crudo anual.

\section{Referencias bibliográficas.}

[1] Rincón Mejía, E.A. Estado del arte de la investigación en energía solar en México. Cuadernos FICA, 1999. 134.

[2] Vélez C., C. Hospitales, Clínicas y Centros de Salud. Guía Sectorial de Producciones más Limpias, 2008. 53.

[3] Bermúdez Rojas, C.R., Ing. and J. García Cuba, Ing., Los calentadores solares como una forma de fuente renovable de energía alternativa, in Ciencias Holguín, 2009: Holguín.

[4] Prats Jové, F. and R. Prats Canós, Mini centrales eléctricas para aguas pasantes, in Centrales eléctricas flotantes (CEFLOT)2007: Barcelona, España. p. 13.

[5] Durham, D., El papel de las energías renovables en España y la Unión Europea en el cumplimiento de los requisitos del Protocolo de Kyoto, 2002, Soluciones energéticas S.A.,: Madrid. p. 116.

[6] Krug, W. Energía eólica. Prácticas introductorias a las energías renovables, , 2001. 47.

[7] Menéndez González, M., Dr., Elementos fundamentales que caracterizan la energía eólica, 2007: La Habana, Cuba. p. 9.

[8] RETScreen International, Clean Energy Proyect Analysis (RETScreen 4), 2008.

[9] Bérriz, L. and M. Álvarez, Manual para el Cálculo y Diseño de Calentadores Solares, ed. Ministerio de Ciencia, Tecnología y Medio Ambiente. 2006, La Habana, Cuba.

[10] González Rodríguez, O.D., Calentador solar de agua con posicionador electrónico, 2010: Jalisco, México.

[11] SODEAN, S.A., Especificaciones técnicas de instalaciones solares térmicas para producción de agua caliente. $1^{\text {ra }}$ ed. 1999, Andalucía, España. 50.

[12] Programa de energía solar de Swisscontact, Manual IV: Sistemas de agua caliente, in Ingeniería Solar. p. 73. 2009.

[13] Programa de energía solar de Swisscontact, Manual II: Sistemas hidráulicos domésticos, in Ingeniería Solar. p. 63. 2009.
[14] Veclima, S.L.U., Estudio de Energía Solar para instalación de A.C.S. en residencia, 2001, solarweb.net: Córdoba, España. p. 17.

[15] RETScreen International, Solar Water Heating Analysis Software (SWH3), 2005.

[16] Proyecto de instalación de energía solar para obtención de agua caliente, 2001: Salamanca, España. 